\title{
Successful Two-Stage Surgical Treatment for Lung Cancer in a Patient with Contralateral Giant Emphysematous Bullae
}

\author{
Yuji Hirami, Katsuhiko Shimizu, Riki Okita, Shinsuke Saisho, Takuro Yukawa, Ai Maeda, \\ Kouichiro Yasuda, Masao Nakata \\ Department of General Thoracic Surgery, Kawasaki Medical School, Okayama, Japan. \\ Email: yhirami@med.kawasaki-m.ac.jp
}

Received August 20 ${ }^{\text {th }}$ 2012; revised September 20 ${ }^{\text {th }}, 2012$; accepted October $20^{\text {th }}, 2012$

\begin{abstract}
A 67-year-old man was referred for further evaluation of an abnormal chest roentgenogram. Computed tomography showed a $40 \times 30 \mathrm{~mm}$ mass in the left upper lobe. A giant bulla occupying about two-thirds of the right thorax was found compressing the adjacent lung parenchyma, shifting the mediastinum to the left. The mass was a primary lung cancer, clinical T2aN0M0, stage IB. Preoperative respiratory function evaluation showed poor pulmonary function, with a forced expiratory volume in 1 second of $1070 \mathrm{ml}$ (29.2\% of predicted). Therefore, we first performed giant bullectomy by video-assisted thoracoscopic surgery. At 1 month after this operation, improvement of the forced expiratory volume in 1 second significantly to $2140 \mathrm{ml}$ (80.1\% of predicted) was observed. Therefore, we performed resection for the tumor. He was discharged after an uneventful postoperative course, and has remained in good condition for 6 months after the operation.
\end{abstract}

Keywords: Giant Emphysematous Bulla; Pulmonary Function; Bullectmy; Lung-Cancer; Surgery

\section{Introduction}

Large emphysematous bullae often compress adjacent lung parenchyma, and cause dyspnea by limiting the ventilator capacity. The most effective treatment, especially for giant bullae occupying more than one-third of the hemithorax, is resection of the bulla, which may be expected to yield immediate functional improvement. Herein, we report a case of successful resection of a lung cancer after initial resection of a contralateral giant emphysematous bulla to improve the deteriorated ventilator capacity.

\section{Case Report}

A 67-year-old man was referred for further evaluation of a left-sided abnormality on the chest roentgenogram pointed out during a medical examination. The patient had been detected to have a giant emphysematous bulla 6 years previously, and had suffered from dyspnea on effort. He was under treatment with inhaled Tiotropium and Salmeterol/Fluticasone. He had smoked 40 cigarettes per day for 40 years, but quit smoking at least 6 years previously. Most of his routine laboratory tests, including the serum CYFRA and ProGRP, were normal, except for an elevated serum CEA level to $8.4 \mathrm{ng} / \mathrm{ml}$. Chest roentgenogram and chest computed tomography (CT) showed a $40 \times 30 \mathrm{~mm}$ mass in the left upper lobe $\mathrm{S} 1+2 \mathrm{a}$ (Figure 1). A giant bulla occupying about two-thirds of the right thorax was found, compressing the adjacent lung parenchyma, and causing a shift of the mediastinum to the left, associated with diffuse emphysema. Positron emission tomography (PET) revealed increased uptake of ${ }^{18} \mathrm{~F}$-fluorodeoxyglucose (FDG) in the mass, with an elevated standard uptake value (SUV) of 19.4. These findings suggested that the mass was probably a primary lung cancer, clinical T2aNOM0, stage IB. Arterial blood gas analysis at rest was almost normal, however, the lung function parameters were impaired with a forced expiratory volume in 1 second $\left(\mathrm{FEV}_{1}\right)$ of $1070 \mathrm{ml}(29.2 \%$ of predicted). The patient experienced dyspnea upon walking a distance of $100 \mathrm{~m}$. Based on these results of preoperative evaluation, we decided to perform resection for the pulmonary tumor after first improving the pulmonary function parameters by performing resection of the giant bulla.

We initially performed bullectomy by video-assisted thoracoscopic surgery (VATS). The giant bulla, originated from the right lower lobe and occupying a large 


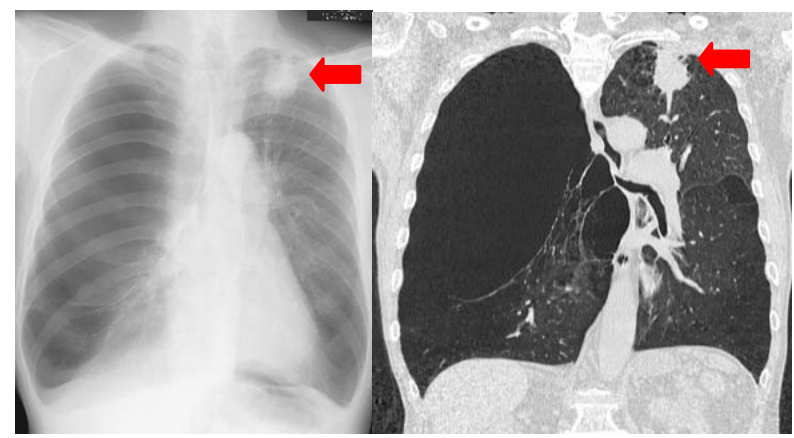

(a)

(b)

Figure 1. (a) Chest roentgenogram showing a mass measuring about 4-cm mass in the left upper lung field (red arrow), and shows hyperlucency of the right upper and middle lung fields; (b) Chest computed tomography (CT) scans showing a $40 \times 30 \mathrm{~mm}$ mass in the left upper lobe $\mathrm{S} 1+$ 2a, with relatively well-defined, but irregular margins, producing a pleural indentation (red arrow). The giant bulla occupies about two-thirds of the right thorax, compressing the adjacent lung parenchyma and producing a shift of the mediastinum to the left, associated with diffuse emphysema.

part of the thoracic cavity, had a relatively thick wall, and was dissected by an automatic stapling device. The suture line was covered with absorption mesh and fixed with fibrin-glue. The operation time was $82 \mathrm{~min}$. After the operation, the patient was extubated in the operating room itself. The thoracic drain was removed on postoperative day 6 , and the patient was discharged after an uneventful course on postoperative day 8. The $\mathrm{FEV}_{1}$ improved significantly to $2140 \mathrm{ml}$ (80.1\% of predicted), and the dyspnea on effort also markedly improved by 1 month after the bullectomy (Figure 2(a)).

Therefore, as the next-stage surgery, we performed wide wedge resection of left upper lobe by VATS for the tumor, because the lung parenchyma on the contralateral side showed severe emphysematous changes, posing a limitation to lobectomy. The pathological diagnosis was primary lung adenocarcinoma, and the pathological stage was T3(pm1)NxM0-stageIIA. The postoperative course was uneventful, and the patient was discharged on postoperative day 7. At 6 months since the operation, the patient remains in good condition, without any evidence of recurrence (Figure 2(b)).

\section{Discussion}

We have presented a case of two-stage surgical treatment for pulmonary carcinoma in a patient with a contralateral giant emphysematous bulla. The reported prevalence rate of lung cancer in patients with bullous disease is $6.1 \%$ [1]. Another report indicated that the prevalence rate of giant bullous disease in patients with lung cancer was $3.9 \%$ [2]. As these patients often have poor pulmonary

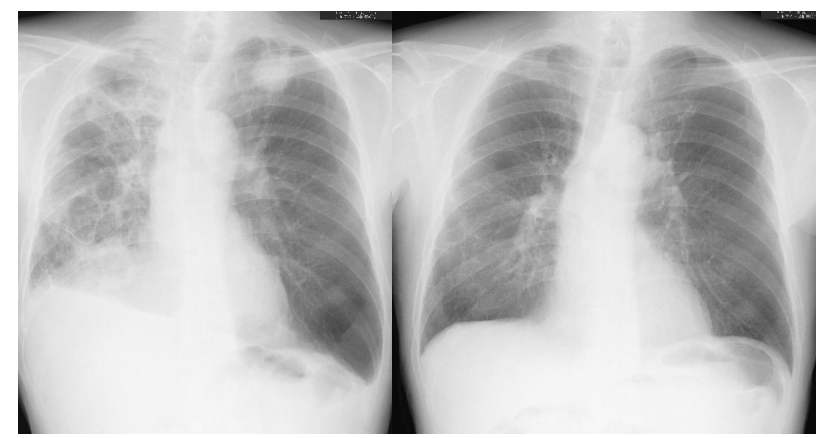

(a)

(b)

Figure 2. (a) At a month after the bullectomy, the chest roentgenogram shows an expanded right lung parenchyma in the hemithorax, with an infiltrative shadow and improvement of the midline shift; (b) At 6 months after the resection for lung cancer, the chest roentgenogram shows a comparative normal lung field.

function, radical pulmonary resection for lung cancer is often difficult.

In general, the indication for bullectomy for a giant bulla is when the bulla occupies half or more of the hemithorax, compresses the normal lung, or has enlarged over a period of years $[3,4]$. Comparison of the preoperative and postoperative (6 months after surgery) pulmonary function tests in patients with giant bullous emphysema has revealed marked clinical improvement and reduction in the severity of dyspnea postoperatively [3,5]. It has also shown that resection of bullae occupying greater than $70 \%$ of the hemithorax produced a doubling of the $\mathrm{FEV}_{1}$ at 1 year, which was sustained at 5 years. Patients with bullae occupying less than $30 \%$ of the hemithorax showed no improvement, with even case of worsening of the $\mathrm{FEV}_{1}$ [4]. Our case was a candidate for bullectomy, because the bulla occupied greater than $70 \%$ of the hemithorax, and moreover compressed the adjacent lung, producing a mediastinal shift to the opposite side.

The problems of this two-stage strategy are the possible complications associated with bullectomy may delay the operation for the lung tumor, and the uncertainty about the degree of improvement of the pulmonary function that can be expected by a mere one month after bullectomy. A previous report [6] showed the results after resection of giant emphysematous bullae in 43 patients, including by VATS in one case. The complications were prolonged air leak lasting for more than 7 days in 53\% of patients, atrial fibrillation in $12 \%$, need for postoperative mechanical ventilation in $9 \%$, and pneumonia in $5 \%$. In regard of the outcome, significant functional improvement was observed, with approximate doubling of the $\mathrm{FEV}_{1}$ by 6 months. We selected bullectomy by VATS in consideration of its being a less- invasive technique and therefore, expectation of an earlier recovery. Bulla surgery by the VATS ap- 
proaches and VATS techniques has gained considerable popularity over recent years [5].

Our patient did not develop any complications of bullectomy and the operation produced an improvement of the $\mathrm{FEV}_{1}$ from $1070 \mathrm{ml}$ (29.2\% of predicted) to 2140 $\mathrm{ml}(80.1 \%$ of predicted) by 1 month after the operation, and also marked symptomatic improvement. In our experience, a point to take care of in relation of giant bullectomy is that complete resection of giant bullae should be avoided, with the surgery performed only with the goal of accomplish to volume reduction. Therefore, the suture line is often above the wall of the giant bulla, which is usually thick as compared to that of small emphysematous bulla. After bullectomy, the thoracic drain must always be placed under a water seal.

To avoid the complications of bullectomy and use less-invasive techniques, bronchoscopic procedures such as transbronchial aspiration needle and one-way endobronchial valves for reduction of giant bullae have been reported $[7,8]$. However, further evaluation of these techniques is needed.

We presented a case of resection for pulmonary carcinoma in a patient with a contralateral giant emphysematous bulla, after resection of the giant bulla to improve the pulmonary function reserve. We wish to emphasize that giant bullectomy could produce significant immediate functional improvement under appropriate conditions.

\section{REFERENCES}

[1] I. L. Stoloff, P. Karnofsky and L. Morgilnea, "The Risk of Lung Cancer in Males with Bullous Disease of the Lung," Archives of Environmental Health, Vol. 22, No. 1, 1971, pp. 163-167.
[2] M. J. Goldstein, G. L. Snider, M. Liberson and R. M. Roske, "Bronchogenic Carcinoma and Giant Bullous Disease," The American Review of Respiratory Disease, Vol. 97, No. 6, 1968, pp. 1062-1070.

[3] A. Palla, M. Desideri, G. Rossi, G. Bardi, D. Mazzanitini, A. Mussi and C. Giuntinic, "Elective Surgery for Giant Bullous Emphysema. A 5-Year Clinical and Functional Follow-Up,” Chest, Vol. 128, No. 4, 2005, pp. 2043-2050. doi:10.1378/chest.128.4.2043

[4] M. X. FitzGerald, P. J. Keelan, D. W. Cugell and E. A. Gaensler, "Long-Term Results of Surgery for Bullous Emphysema,” The Journal of Thoracic and Cardiovascular Surgery, Vol. 68, No. 4, 1974, pp. 566-587.

[5] G. F. Menconi, F. M. Antonietta Melfi, A. Mussi, A. Palla, M. C. Ambrogi and C. A. Angeletti, "Treatment by VATS of Giant Bullous Emphysema: Results,” European Journal of Cardio-Thoracic Surgery, Vol. 13, No. 1, 1998, pp. 66-70. doi:10.1016/S1010-7940(97)00294-7

[6] P. H. Schipper, B. F. Meyers, R. J. Battafarano, T. J. Guthrie, G. A. Patterson and J. D. Cooper, "Outcomes after Resection of Giant Emphysematous Bullae,” The Annals of Thoracic Surgery, Vol. 78, No. 3, 2004, pp. 976-982. doi:10.1016/j.athoracsur.2004.04.005

[7] P. Bhattacharyya, D. Sarkar, S. Nag, S. Ghosh and S. Roychoudhury, "Transbronchial Decompression of Emphysematous Bullae: A New Therapeutic Approach,” The European Respiratory Journal, Vol. 29, No. 5, 2007, pp. 1003-1006. doi:10.1183/09031936.00030106

[8] M. Santini, A. Fiorelli, G. Vicidomini, V. Giuseppe Di Crescenzo, G. Messina and P. Laperuta, "Endobronchial Treatment of Giant Emphysematous Bullae with OneWay Valves: A New Approach for Surgically Unfit Patients," The European respiratory Journal, Vol. 40, No. 6, 2011, pp. 1425-1431. 